\title{
Pontryagin's Maximum Principle for the Optimal Control Problems with Multipoint Boundary Conditions
}

\author{
M. J. Mardanov ${ }^{1}$ and Y. A. Sharifov ${ }^{2,3}$ \\ ${ }^{1}$ Institute of Mathematics and Mechanics of ANAS, 9 B. Vahabzadeh Street, 1141 Baku, Azerbaijan \\ ${ }^{2}$ Institute of Control Systems of ANAS, 9 B. Vahabzadeh Street, 1141 Baku, Azerbaijan \\ ${ }^{3}$ Baku State University, 23 Z. Khalilov Street, 1148 Baku, Azerbaijan \\ Correspondence should be addressed to Y. A. Sharifov; sharifov22@rambler.ru
}

Received 5 August 2014; Revised 29 September 2014; Accepted 30 September 2014

Academic Editor: Allaberen Ashyralyev

Copyright (c) 2015 M. J. Mardanov and Y. A. Sharifov. This is an open access article distributed under the Creative Commons Attribution License, which permits unrestricted use, distribution, and reproduction in any medium, provided the original work is properly cited.

Optimal control problem with multipoint boundary conditions is considered. Sufficient conditions for the existence and uniqueness of the solution of boundary value problem for every fixed admissible control are obtained. First order increment formula for the functional is derived. Pontryagin's maximum principle is proved by using the variations of admissible control.

\section{Introduction}

Boundary value problems appear in a large field of sciences to describe physical, biological, and chemical phenomena and several practically important problems lead to multipoint boundary value problems. Some examples are given in the area of elasticity and on the effects of soil settlement [15]. For boundary value problems with multipoint boundary conditions and comments on their importance, we refer the reader to the papers [6-11] and the references therein.

Pontryagin's maximum principle is the first order necessary optimality condition and occupies a special place in theory of optimal processes. Originally the maximum principle was proved for the Cauchy system of ordinary differential equations [12]. Later on this result was carried over the most complex objects described by the equations with a delay, integral equations, partial equations, stochastic equations, and so forth (see, e.g., $[13,14]$ and the references therein).

At present, there exists a great amount of work devoted to derivation of necessary optimality conditions of first and second orders for the systems with local conditions (see [12, 14-19] and the references therein).

Recently, the optimal control problems with nonlocal conditions are intensively investigated. In the papers $[13,20-24]$ the necessary optimality conditions for optimal control problems described by the systems of ordinary differential equations with nonlocal conditions were obtained. In these papers the nonlocal conditions contain two-point and integral boundary conditions.

It is known that the solution of problems of mechanics and control processes is reduced to multipoint boundary value problems. The constructive sufficient existence and uniqueness conditions and also the methods of numerical solution of such boundary value problems were studied in [69].

In the present paper, Pontryagin's maximum principle for optimal control problems for the ordinary differential equations with multipoint boundary conditions is proved. Since in optimal control problems with multipoint boundary conditions the solution of the associated system has discontinuities of the first kind of inner points, the direct applications of the solution methods of two-point boundary value problems to optimal control problems with multipoint boundary conditions are impossible.

The paper is organized as follows. First, we give the statement of the problem. Second, theorems on existence and uniqueness of the solution of problem (1)-(3) are established under some sufficient conditions on the nonlinear terms. 
Third, the first order increment formula for the functional is presented and Pontryagin's maximum principle is provided.

\section{Problem Statement}

Let the controlled process on a fixed time interval $[0, T]$ be described by a system of differential equations

$$
\frac{d x}{d t}=f(t, x, u)
$$

with multipoint boundary conditions

$$
\sum_{j=0}^{N} B_{j} x\left(t_{j}\right)=C
$$

where $x(t) \in R^{n} ; f(t, x, u)$ is the given $u$ dimensional vectorfunction; $C \in R^{n}$ is the given constant vector; $0=t_{0}<t_{1}<$ $\cdots<t_{N}=T$ are fixed points; $u(t)$ is the $r$ dimensional and bounded vector of control actions with the values from the nonempty, bounded set $U$; that is,

$$
u(t) \in U \subset R^{r}, \quad t \in[0, T] .
$$

It is required to minimize the functional

$$
J(u)=\varphi(x(0), x(T))+\int_{0}^{T} F(t, x, u) d t
$$

subject to (1)-(3).

Here we assumed that the functions $f(t, x, u), F(t, x, u)$, and $\varphi(x, y)$ are continuous over the set of arguments and have bounded partial derivatives with respect to the arguments $x$ and $y$. Under the solution of problem (1)-(3) that corresponds to the fixed admissible control $u(t)$ we take the function $x(t)$ : $[0, T] \rightarrow R^{n}$ absolutely continuous on the interval $[0, T]$.

Denote by $C\left([0, T], R^{n}\right)$ a space of continuous functions on the interval $[0, T]$ with the values from $R^{n}$. Obviously, such a space is Banach with the norm

$$
\|x\|_{C[0, T]}=\max _{[0, T]}|x(t)|
$$

where $|\cdot|$ is the norm $R^{n}$.

The admissible process $\{u(t), x(t, u)\}$, being the solution of problem (1)-(4), that is, delivering minimum to the functional (4) under restrictions (1)-(3), will be called an optimal process and $u(t)$ an optimal control.

\section{Existence of Solutions of Boundary Value Problem (1)-(3)}

Introduce the following conditions.

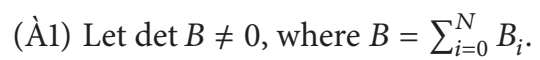

(À2) The function $f:[0, T] \times R^{n} \times R^{n} \rightarrow R^{n}$ is continuous and there exists a constant $K \geq 0$ such that

$$
\begin{array}{r}
|f(t, x, u)-f(t, y, u)| \leq K|x-y|, \\
t \in[0, T], \quad x, y \in R^{n}, \quad u \in U .
\end{array}
$$

$M(t, s)$ is a piecewise matrix such that $t_{k-1} \leq s<t_{k},(k=$ $1,2, \ldots, N)$ :

$$
M(t, s)= \begin{cases}B^{-1} \sum_{i=0}^{k-1} B_{i}, & \text { if } s<t, \\ -B^{-1} \sum_{i=k}^{N} B_{i}, & \text { if } t \leq s .\end{cases}
$$

Theorem 1. Let condition (À1) be fulfilled. The function $x(\cdot) \in$ $C\left([0, T], R^{n}\right)$ is an absolute continuous solution of problem (1)(3) if and only if

$$
x(t)=B^{-1} C+\int_{0}^{T} M(t, s) f(s, x(s), u(s)) d s,
$$

where the matrix function $M(t, s)$ is determined by equality (7).

Proof. Let the function $x=x(t)$ be a solution of (1). Then for $t \in(0, T)$ the formula is valid:

$$
x(t)=x(0)+\int_{0}^{t} f(s, x(s), u(s)) d s,
$$

where $x(0)$ is an arbitrary constant vector. In order to determine $x(0)$ the required function defined by equality (9) satisfies condition (2):

$$
\sum_{i=0}^{N} B_{i} x(0)=C-\sum_{i=0}^{N} B_{i} \int_{0}^{t_{i}} f(t, x(t), u(t)) d s .
$$

Since, according to condition (À1), $\operatorname{det} B \neq 0$, then it follows from equality (10) that

$$
x(0)=B^{-1} C-B^{-1} \sum_{i=0}^{N} B_{i} \int_{0}^{t_{i}} f(s, x(s), u(s)) d s,
$$

which may be rewritten in the form

$$
x(0)=B^{-1} C-B^{-1} \sum_{k=1}^{N}\left[\sum_{i=k}^{N} B_{i} \int_{t_{k-1}}^{t_{k}} f(s, x(s), u(s)) d s\right] \text {. }
$$

Now taking into account the value of $x(0)$ determined by equality (12) in (9) we get

$$
\begin{aligned}
x(t)= & B^{-1} C-B^{-1} \sum_{k=1}^{N}\left[\sum_{i=k}^{N} B_{i} \int_{t_{k-1}}^{t_{k}} f(s, x(s), u(s)) d s\right] \\
& +\int_{0}^{t} f(s, x(s), u(s)) d s .
\end{aligned}
$$

Obviously, for $t_{k-1} \leq t<t_{k}$, we can write equality (13) in equivalent form:

$$
\begin{aligned}
x(t) & =B^{-1} C+\int_{0}^{t}\left[E-B^{-1} \sum_{i=k}^{N} B_{i}\right] f(s, x(s), u(s)) d s \\
& =\int_{t}^{t_{k}}\left(B^{-1} \sum_{i=k}^{N} B_{i}\right) f(s, x(s), u(s)) d s .
\end{aligned}
$$

(À3) $L=K T M<1$, where $M=\max _{0 \leq t, s \leq T}\|M(t, s)\|$. 
So

$$
E-B^{-1} \sum_{i=k}^{N} B_{i}=B^{-1}\left(\sum_{i=0}^{N} B_{i}-\sum_{i=k}^{N} B_{i}\right)=B^{-1} \sum_{i=0}^{k-1} B_{i}
$$

holds; then by using (7) we can rewrite equality (14) in the following equivalent form:

$$
x(t)=B^{-1} C+\int_{0}^{T} M(t, s) f(s, x(s), u(s)) d s .
$$

Thus, it is shown that boundary value problem (1)-(3) may be rewritten in equivalent integral form (8). By direct calculation we can show that the solution of integral equation (8) is a solution of boundary value problem (1)-(3).

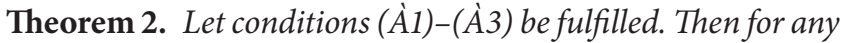
$C \in R^{n}$ and any fixed admissible control, boundary value problem (1)-(3) has the unique solution satisfying the integral equation (8).

Proof. Let $C \in R^{n}$ and $u(\cdot) \in U$ be fixed. Let us consider the mapping $P: C\left([0, T], R^{n}\right) \rightarrow C\left([0, T], R^{n}\right)$ determined according to the rule:

$$
(P x)(t)=B^{-1} C+\int_{0}^{T} M(t, s) f(s, x(s), u(s)) d s .
$$

Obviously, the fixed points of the operator $P$ are a solution of boundary value problem (1)-(2). Using the Banach method of contractive operators we show that the operator $P$ determined by equality (17) has a fixed point. For any $v, \omega \in$ $C\left([0, T], R^{n}\right)$ we have

$$
\begin{aligned}
& |(P v)(t)-(P \omega)(t)| \\
& \leq \int_{0}^{T}|M(t, s)| \cdot|f(s, v(s), u(s))-f(s, \omega(s), u(s))| d s \\
& \leq K T M\|v(\cdot)-\omega(\cdot)\|_{C[0, T]}
\end{aligned}
$$

or

$$
\|(P v)(t)-(P \omega)(t)\|_{C[0, T]} \leq K T M\|v(\cdot)-\omega(\cdot)\|_{C[0, T]} .
$$

Here taking into account condition (À3) we get that the operator $P$ has the unique fixed point in (17). This shows that integral equation (8) has the unique solution and therefore the equivalent boundary value problem (1)-(3) also has a unique solution. Theorem 2 is proved.

\section{Increment Formula for the Functional}

The increment method is one of the simplest ones among the methods for proving the maximum principle. In order to obtain the necessary conditions for optimality, we will use the standard procedure (see, e.g., [16]).

Let $u=u(t)$ and $\tilde{u}(t)=u(t)+\Delta u(t), t \in[0, T]$, be two admissible controls and let $x(t), \tilde{x}(t)=x(t)+\Delta x(t)$, $t \in[0, T]$, be appropriate trajectories. Then, obviously, $\Delta x(t)$ is the solution of the following boundary value problem:

$$
\begin{gathered}
\Delta \dot{x}(t)=\Delta f(t, x, u), \quad t \in[0, T], \\
\sum_{i=0}^{N} B_{i} \Delta x\left(t_{i}\right)=0 .
\end{gathered}
$$

Here $\Delta f(t, x, u)=f(t, \tilde{x}, \tilde{u})-f(t, x, u)$ are the denotations of total increment of the function $f(t, x, u)$. We can write the increment of functional (4) in the form

$$
\begin{aligned}
\Delta J(u)= & J(\bar{u})-J(u)=\Delta \varphi(x(0), x(T)) \\
& +\int_{0}^{T} \Delta F(t, x, u) d t .
\end{aligned}
$$

Let $\psi(t) \in R^{n}$ be an arbitrary nontrivial vector-function and let $x \in R^{n}$ be a scalar vector. Then we can rewrite the increment of functional (4) in the form

$$
\begin{aligned}
\Delta J(u)= & J(\widetilde{u})-J(u)=\Delta \varphi(x(0), x(T)) \\
& +\int_{0}^{T} F(t, x, u) d t \\
& +\int_{0}^{T}\langle\psi(t), \Delta \dot{x}(t)-\Delta f(t, x, u)\rangle d t \\
& +\left\langle\lambda, \sum_{i=0}^{N} B_{i} \Delta x\left(t_{i}\right)\right\rangle .
\end{aligned}
$$

After some standard operators usually used in deriving necessary optimality conditions of the first order, for the increments formula we get the following equality:

$$
\begin{aligned}
\Delta J & (u) \\
= & -\int_{0}^{T} \Delta_{\tilde{u}} H(t, \psi, x, u) d t \\
& -\int_{0}^{T}\left\langle\Delta_{\widetilde{u}} \frac{\partial H(t, \psi, x, u)}{\partial x}, \Delta x(t)\right\rangle d t \\
& +\int_{0}^{T}\left\langle\dot{\psi}(t)+\frac{\partial H(t, \psi, x, u)}{\partial x}, \Delta x(t)\right\rangle d t \\
& +\left\langle-\psi(0)+B_{0}^{\prime} \lambda+\frac{\partial \varphi}{\partial x(0)}, \Delta x(0)\right\rangle \\
& +\left\langle\psi(T)+B_{N}^{\prime} \lambda+\frac{\partial \varphi}{\partial x(T)}, \Delta x(T)\right\rangle \\
& +\left\langle\sum_{i=1}^{N-1}\left[-\psi\left(t_{i}+0\right)+\psi\left(t_{i}-0\right)+B_{i}^{\prime} \lambda\right], \Delta x\left(t_{i}\right)\right\rangle+\eta_{\bar{u}} \\
& \eta_{\bar{u}}=o_{\varphi}(\|\Delta x(0)\|,\|\Delta x(T)\|)-\int_{0}^{T} o_{H}(\Delta\|x(t)\|) d t
\end{aligned}
$$

where $H(t, \psi, x, u)=\langle\psi(t), f(t, x, u)\rangle-F(t, x, u)$. 
Now assume that the unknown vector-function $\psi(t) \in R^{n}$ and the $\lambda$ scalar vector is a solution of the following boundary value problem:

$$
\begin{gathered}
\dot{\psi}(t)=-\frac{\partial H(t, \psi, x, u)}{\partial x}, \\
t \in[0, T], \quad t \neq t_{i}, \quad i=1,2, \ldots, N-1, \\
\psi(0)=B_{0}^{\prime} \lambda+\frac{\partial \varphi}{\partial x(0)}, \quad \psi(T)=-B_{N}^{\prime} \lambda-\frac{\partial \varphi}{\partial x(T)}, \\
\psi\left(t_{i}+0\right)-\psi\left(t_{i}-0\right)=B_{i}^{\prime} \lambda, \quad i=1,2, \ldots, N-1 .
\end{gathered}
$$

The difference-differential (24)-(26) boundary value problem is called an adjoint problem in parametric form since it conditions the unknown parameter $\lambda$. From the adjoint system (24)-(25) it is seen that the solution of this system at the points $t=t_{i},(i=1,2, \ldots, N-1)$, has the first order discontinuities. This is the essential peculiarity of multipoint boundary conditions.

From condition (À1) and from the system (24)-(25) we can exclude the unknown vector $\lambda$. Indeed, from equality (25) we have

$$
\begin{aligned}
\sum_{i=0}^{N} B_{i}^{\prime} \lambda= & \psi(0)-\frac{\partial \varphi}{\partial x(0)}-\psi(T)-\frac{\partial \varphi}{\partial x(T)} \\
& +\sum_{i=1}^{N-1}\left(\psi\left(t_{i}+0\right)-\psi\left(t_{i}-0\right)\right) .
\end{aligned}
$$

Hence

$$
\begin{array}{r}
\lambda=\left(B^{\prime}\right)^{-1}\left[\psi(0)-\frac{\partial \varphi}{\partial x(0)}-\psi(T)-\frac{\partial \varphi}{\partial x(T)}\right. \\
\left.+\sum_{i=1}^{N-1}\left(\psi\left(t_{i}+0\right)-\psi\left(t_{i}-0\right)\right)\right] .
\end{array}
$$

Now take into account the found value of $\lambda$ expressed in (26) in equalities (25) and (26). Then equalities (25) and (26) take the form

$$
\begin{aligned}
& \psi(0) \\
& =B_{0}^{\prime}\left(B^{\prime}\right)^{-1}\left[\psi(0)-\frac{\partial \varphi}{\partial x(0)}-\psi(T)-\frac{\partial \varphi}{\partial x(T)}\right. \\
& \\
& \left.+\sum_{i=1}^{N-1}\left(\psi\left(t_{i}+0\right)-\psi\left(t_{i}-0\right)\right)\right]+\frac{\partial \varphi}{\partial x(0)},
\end{aligned}
$$

$$
\begin{aligned}
& \psi(T) \\
& =B_{N}^{\prime}\left(B^{\prime}\right)^{-1}\left[\psi(0)-\frac{\partial \varphi}{\partial x(0)}-\psi(T)-\frac{\partial \varphi}{\partial x(T)}\right. \\
& \left.+\sum_{i=1}^{N-1}\left(\psi\left(t_{i}+0\right)-\psi\left(t_{i}-0\right)\right)\right]-\frac{\partial \varphi}{\partial x(T)}, \\
& \psi\left(t_{i}+0\right)-\psi\left(t_{i}-0\right) \\
& =B_{i}^{\prime}\left(B^{\prime}\right)^{-1} \\
& \times\left[\psi(0)-\frac{\partial \varphi}{\partial x(0)}-\psi(T)-\frac{\partial \varphi}{\partial x(T)}\right. \\
& \left.+\sum_{i=1}^{N-1}\left(\psi\left(t_{i}+0\right)-\psi\left(t_{i}-0\right)\right)\right] .
\end{aligned}
$$

Taking into account equalities (24) and (25) in (23), we get the final form for the increment of the functional

$$
\begin{aligned}
\Delta J(u)= & -\int_{0}^{T} \Delta_{\tilde{u}} H(t, \psi, x, u) d t \\
& -\int_{0}^{T}\left\langle\Delta_{\tilde{u}} \frac{\partial H(t, \psi, x, u)}{\partial x}, \Delta x(t)\right\rangle d t+\eta_{\tilde{u}} .
\end{aligned}
$$

\section{Pontryagin's Maximum Principle}

At different proofs of the maximum principle, the needleshaped variation plays one of the main parts. We choose the "perturbed" control $\widetilde{u}(t)$ in the special way:

$$
\widetilde{u}(t)= \begin{cases}u(t), & t \notin[\theta, \theta+\varepsilon), \\ v, & t \in[\theta, \theta+\varepsilon),\end{cases}
$$

where the parameters of the needle-shaped variation satisfy the following conditions. $\theta \in[0, T]$ is a regular point of the control $u(t), \varepsilon>0, \theta+\varepsilon<T, v \in U$. For any $\theta, \varepsilon, v$ satisfying the enumerated conditions, the control $\bar{u}(t)$ is admissible.

The traditional form of necessary optimality conditions will follow from increments formula (30) if we show that on the needle-shaped variation $\widetilde{u}(t)=u_{\varepsilon}(t)$ the increment of phase states $\Delta_{\varepsilon} x(t)$ is of order $\varepsilon$. This will follow from

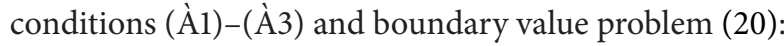

$$
\begin{aligned}
\Delta x(t)= & \int_{0}^{T} M(t, s)[f(s, x+\Delta x, \tilde{u})-f(s, x, \tilde{u})] d s \\
& +\int_{0}^{T} M(t, s) \Delta_{\tilde{u}} f(s, x, u) d s .
\end{aligned}
$$

From (32) we get

$$
\|\Delta x(t)\| \leq(1-L)^{-1} M \int_{0}^{T}\left\|\Delta_{\tilde{u}} f(t, x, u)\right\| d t .
$$


If in inequality we take $\widetilde{u}(t)=u_{\varepsilon}(t)$, we have

$$
\left\|\Delta_{\varepsilon} x(t)\right\| \leq \widetilde{L} \varepsilon, \quad t \in[0, T], \widetilde{L}=\text { const }>0 .
$$

Estimation (33) shows that, for $\widetilde{u}(t)=u_{\varepsilon}(t)$,

$$
\begin{aligned}
\int_{\theta}^{\theta+\varepsilon} & \left\langle\Delta_{v} \frac{\partial H(t, \psi, x, u)}{\partial x}, \Delta_{\varepsilon} x(t)\right\rangle d t \\
& +\eta_{u_{\varepsilon}}\left(\left\|\Delta_{\varepsilon} x(t)\right\|\right)=o(\varepsilon)
\end{aligned}
$$

where $\Delta_{\varepsilon} x(t)=x\left(t, u_{\varepsilon}\right)-x(t, u) \sim \varepsilon$.

Use the increment formula (30) and the property of the needle-shaped variation. Then

$$
\Delta J(u)=-\int_{\theta}^{\theta+\varepsilon} \Delta_{v} H(t, \psi, x, u) d t+o(\varepsilon) .
$$

Since the point $t=\theta$ is a regular point of the control $u=$ $u(t)$, from the Taylor formula it follows that

$$
\begin{array}{r}
\Delta J(u)=-\Delta_{v} H(\theta, \psi(\theta), x(\theta), u(\theta)) \varepsilon+o(\varepsilon), \\
v \in U, \quad \theta \in[0, T) .
\end{array}
$$
(37).

Pontryagin's maximum principle follows from formula

Theorem 3 (maximum principle). Let the admissible process $u^{0}(t), x^{0}\left(t, u^{0}\right)$ be optimal in problem (1)-(4) and let $\psi^{0}(t)$ be a solution of conjugated problem (24)-(25) calculated on optimal process. Then for all $t \in[0, T]$ the following equality is fulfilled:

$$
\max _{v \in U} H\left(t, \psi^{0}(t), x^{0}(t), v\right)=H\left(t, \psi^{0}(t), x^{0}(t), u^{0}(t)\right) .
$$

Corollary 4. If in the optimal control problem the function $f$ is linear with respect to $(x, u)$ and the functions $\varphi, F$ are convex with respect to $x(0), x(T)$ and $x(t)$, respectively, then the maximum principle is necessary and sufficient for optimality. This fact follows from increment formula (30). Indeed, in this case,

$$
\begin{aligned}
\Delta J(u)= & -\int_{0}^{T} \Delta_{\bar{u}} H(t, \psi, x, u) d t+o_{\varphi}(\|\Delta x(0)\|,\|\Delta x(T)\|) \\
& +\int_{0}^{T} o_{F}(\|\Delta x(t)\|) d t .
\end{aligned}
$$
0 .

Since the functions $\varphi$ and $F$ are convex, then $o_{\varphi} \geq 0, o_{F} \geq$

Other Optimality Conditions. In this item we suppose that the function $f(t, x, u)$ is differentiable and the set $U$ is convex. Then from Theorem 3 we get the following theorem.
Theorem 5 (differential principle of maximum). Let the process $\left(u^{0}(t), x^{0}\left(t, u^{0}\right)\right), t \in[0, T]$, be optimal in problem (1)(4) and let $\psi^{0}(t)$ be an appropriate solution of adjoint problem (24)-(26). Then,

$$
\begin{aligned}
& \left\langle\frac{\partial H\left(t, \psi^{0}(t), x^{0}(t), u^{0}(t)\right)}{\partial u}, u^{0}(t)\right\rangle \\
& \quad=\max _{v \in U}\left\langle\frac{\partial H\left(t, \psi^{0}(t), x^{0}(t), u^{0}(t)\right)}{\partial u}, v\right\rangle .
\end{aligned}
$$

Proof. Suppose the contrary. Let there exist $\theta \in[0, T], v \in U$, $\alpha>0$, such that

$$
\begin{aligned}
& \left\langle\frac{\partial H\left(\theta, \psi^{0}(\theta), x^{0}(\theta), u^{0}(\theta)\right)}{\partial u}, u^{0}(\theta)\right\rangle \\
& \quad=\left\langle\frac{\partial H\left(\theta, \psi^{0}(\theta), x^{0}(\theta), u^{0}(\theta)\right)}{\partial u}, v\right\rangle-\alpha .
\end{aligned}
$$

Let $0 \leq \varepsilon \leq 1$. Construct the vector

$$
v(\varepsilon)=u^{0}(\theta)+\varepsilon\left(v-u^{0}(\theta)\right) .
$$

Since the set $U$ is convex, then $v(\varepsilon) \in U$. From maximum principle (38) and equality (41) it follows that the inequality

$$
\begin{aligned}
0 \geq & \Delta_{v(u)} H\left(\theta, \psi^{0}(\theta), x^{0}(\theta), u^{0}(\theta)\right) \\
= & \left\langle\frac{\partial H\left(\theta, \psi^{0}(\theta), x^{0}(\theta), u^{0}(\theta)\right)}{\partial u}, v(\varepsilon)-u^{0}(\theta)\right\rangle \\
& +o\left(\left\|v(\varepsilon)-u^{0}(\theta)\right\|\right)=\alpha \varepsilon+o(\varepsilon)
\end{aligned}
$$

is valid for all rather small $\varepsilon$, which is impossible, since by supposition $\alpha>0$. Indeed, the right part of (43) for rather small $\varepsilon>0$ is positive. The theorem is proved.

Note that condition (40) for verification is simpler than condition (38) by virtue of linearity of the right hand side of (43). However, assumptions on convexity of $U$ and differentiability of the function $f(t, x, u)$ with respect to $u$ contract the application of condition (41).

Note that there exist optimal control problems for which condition (40) is valid, and the maximum principle gives no information. This determines the value of the differential principle of maximum.

The following theorem follows from the maximum principle.

Theorem 6 (stationary state principle). Let $U \subset R^{n}$ be an open set. Then at each $t \in[0, T]$ the optimal control delivers the stationary value to the function $H(t, \psi, x, u)$; that is,

$$
\frac{\partial H\left(t, \psi^{0}(t), x^{0}(t), u^{0}(t)\right)}{\partial u}=0 .
$$




\section{Discussion of the Obtained Results}

In this paper different necessary optimality conditions of first order were obtained for optimal control problems with multipoint boundary conditions. This problem is rather general and contains different special cases.

(i) The first case is the Cauchy problem (in this case $N=$ 0 and $B_{0}$ is a unit matrix).

(ii) The second case is the problem with two-point boundary conditions (in this case $N=1$ ).

(iii) Each equation of (1) has its initial condition; that is, dimension of the vector $x$ equals $N+1$ and $B_{i}=\left(B_{j k}^{i}\right)$ $(i=0,1, \ldots, N ; j, k=1,2, \ldots, N+1)$ and

$$
B_{j k}^{i}=\left\{\begin{array}{l}
1, \quad j=i+1, k=k(i), \\
0 .
\end{array}\right.
$$

Here $(k(0), k(1), \ldots, k(N))$ is some permutation $(1,2, \ldots, N+1)$.

\section{Conflict of Interests}

The authors declare that there is no conflict of interests regarding the publication of this paper.

\section{References}

[1] J. Prescott, Applied Elasticity, Dover Publications, New York, NY, USA, 1961.

[2] S. P. Timoshenko, Theory of Elastic Stability, McGraw-Hill, New York, NY, USA, 1961.

[3] E. H. Mansfield, The Bending and Stretching of Plates, vol. 6 of International Series of Monographs on Aeronautics and Astronautics, Pergamon Press, New York, NY, USA, 1964.

[4] E. Dulacska, Soil Settlement Effects Buildings, Development Getechn. Engrg., vol. 69, Elsevier, Amsterdam, The Netherlands, 1992.

[5] A. Dhamacharen and K. Chompuvised, "An efficient method for solving multipoint equation boundary value problems," World Academy of Science, Engineering and Technology, vol. 75, pp. 6165, 2013.

[6] M. Urabe, "An existence theorem for multi-point boundary value problems," Funkcialaj Ekvacioj. Serio Internacia, vol. 9, pp. 43-60, 1966.

[7] A. Ashyralyev and O. Yildirim, "On multipoint nonlocal boundary value problems for hyperbolic differential and difference equations," Taiwanese Journal of Mathematics, vol. 14, no. 1, pp. 165-194, 2010.

[8] P. W. Eloe and J. Henderson, "Multipoint boundary value problems for ordinary differential systems," Journal of Differential Equations, vol. 114, no. 1, pp. 232-242, 1994.

[9] J. R. Graef and L. Kong, "Solutions of second order multi-point boundary value problems," Mathematical Proceedings of the Cambridge Philosophical Society, vol. 145, no. 2, pp. 489-510, 2008.

[10] V. A. Il'in and E. I. Moiseev, "Nonlocal boundary value problem of the first kind for a strum-Lowville operator in its differential and difference aspects," Differential Equations, vol. 23, pp. 803810, 1987.
[11] V. A. Il'in and E. I. Moiseev, "Nonlocal boundary value problem of the first kind for a Strum-Lowville operator," Differential Equations, vol. 23, pp. 979-987, 1987.

[12] L. Pontryagin, V. Boltyanski, R. Gamkrelidze, and E. Mishchenko, Mathematical Theory of Optimal Processes, Wiley, Chichester, UK, 1962.

[13] Y. A. Sharifov, "Optimality conditions in problems of control over systems of impulsive differential equations with nonlocal boundary conditions," Ukrainian Mathematical Journal, vol. 64, no. 6, pp. 958-970, 2012.

[14] R. Gabasov and F. M. Kirillova, Singular Optimal Controls, Nauka, Moscow, Russia, 1973, (Russian).

[15] F. P. Vasil'ev, Optimization Methods, Factorial Press, Moscow, Russia, 2002.

[16] O. V. Vasiliev, Optimization Methods (Advanced Series in Mathematical Science and Engineering), vol. 5, World Federation Publishers Company, Atlanta, Ga, USA, 1996.

[17] A. J. Krener, "The high order maximal principle and its application to singular extremals," SIAM Journal on Control and Optimization, vol. 15, no. 2, pp. 256-293, 1977.

[18] H. J. Kelley, R. E. Kopp, and H. G. Moyer, "Singular extremals," in Topics in Optimization, G. Leitmann, Ed., pp. 63-101, Academic Press, New York, NY, USA, 1967.

[19] M. J. Mardanov, K. B. Mansimov, and T. K. Melikov, Investigation of Singular Controls and Second Order Necessary Optimality Conditions in Systems with Delay, Elm, Baku, Azerbaijan, 2013.

[20] Y. A. Sharifov and N. B. Mamedova, "On second-order necessary optimality conditions in the classical sense for systems with nonlocal conditions," Differential Equations, vol. 48, no. 4, pp. 605-608, 2012.

[21] M. F. Mekhtiyev, S. I. Djabrailov, and Y. A. Sharifov, "Necessary optimality conditions of second order in classical sense in optimal control problems of three-point conditions," Journal of Automation and Information Sciences, vol. 42, no. 3, pp. 47-57, 2010.

[22] O. Vasilieva and K. Mizukami, "Optimality criterion for singular controllers: linear boundary conditions," Journal of Mathematical Analysis and Applications, vol. 213, no. 2, pp. 620-641, 1997.

[23] A. Ashyralyev and Y. A. Sharifov, "Optimal control problems for impulsive systems with integral boundary conditions," Electronic Journal of Differential Equations, vol. 2013, no. 80, pp. 1-11, 2013.

[24] A. R. Safari, M. F. Mekhtiyev, and Y. A. Sharifov, "Maximum principle in the optimal control problems for systems with integral boundary conditions and its extension," Abstract and Applied Analysis, vol. 2013, Article ID 946910, 9 pages, 2013. 


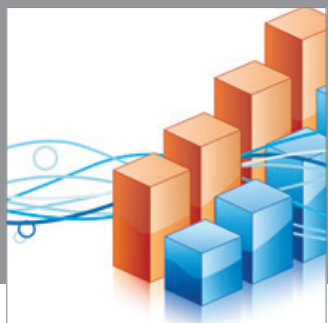

Advances in

Operations Research

mansans

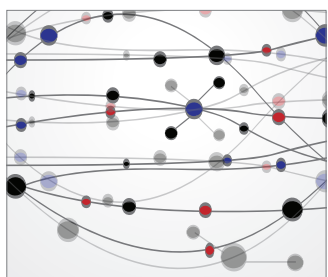

The Scientific World Journal
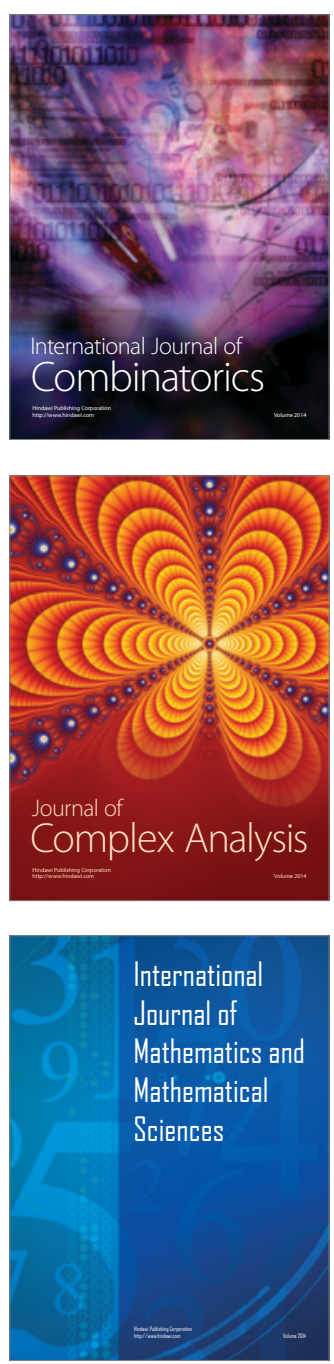
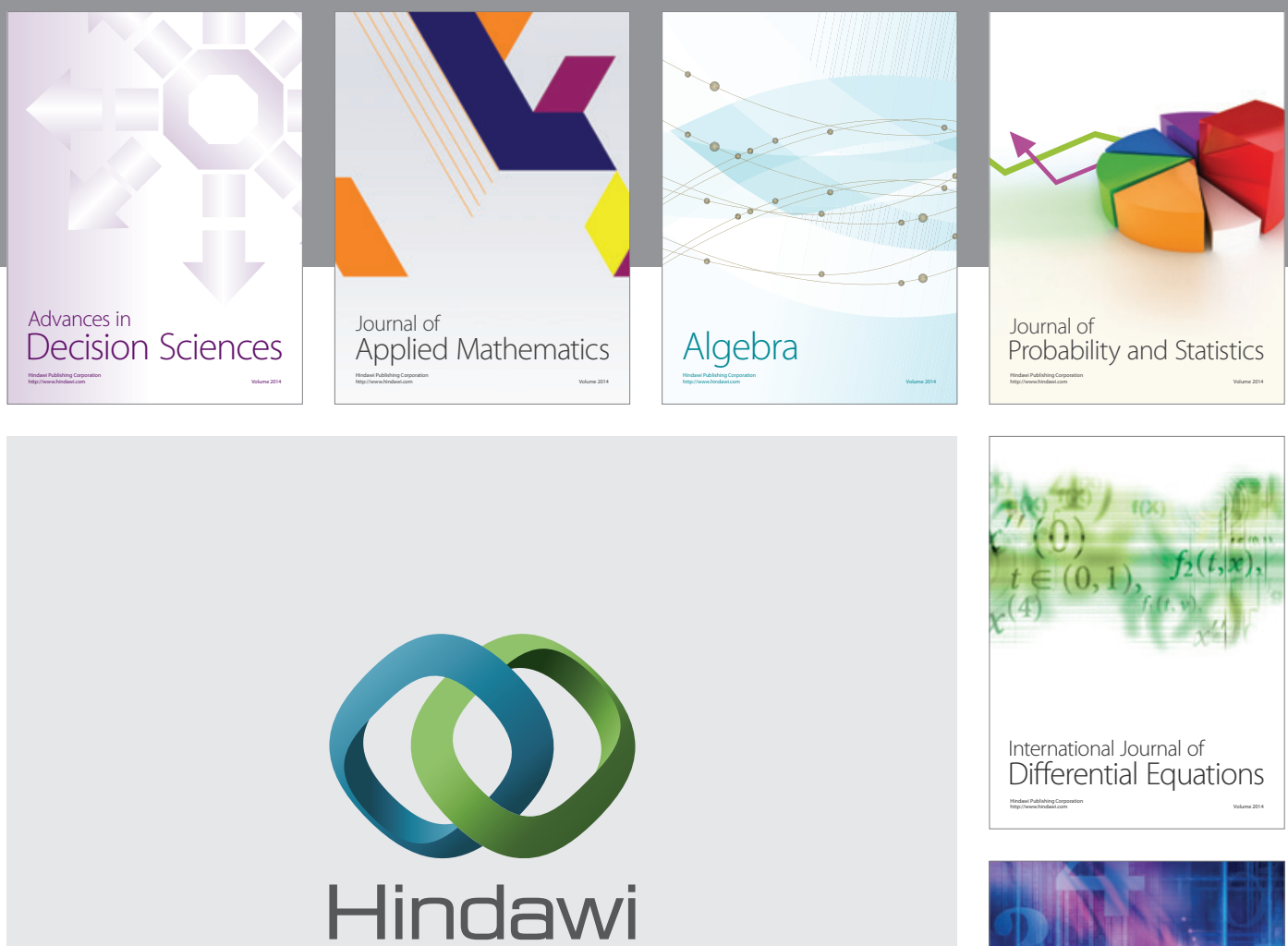

Submit your manuscripts at http://www.hindawi.com
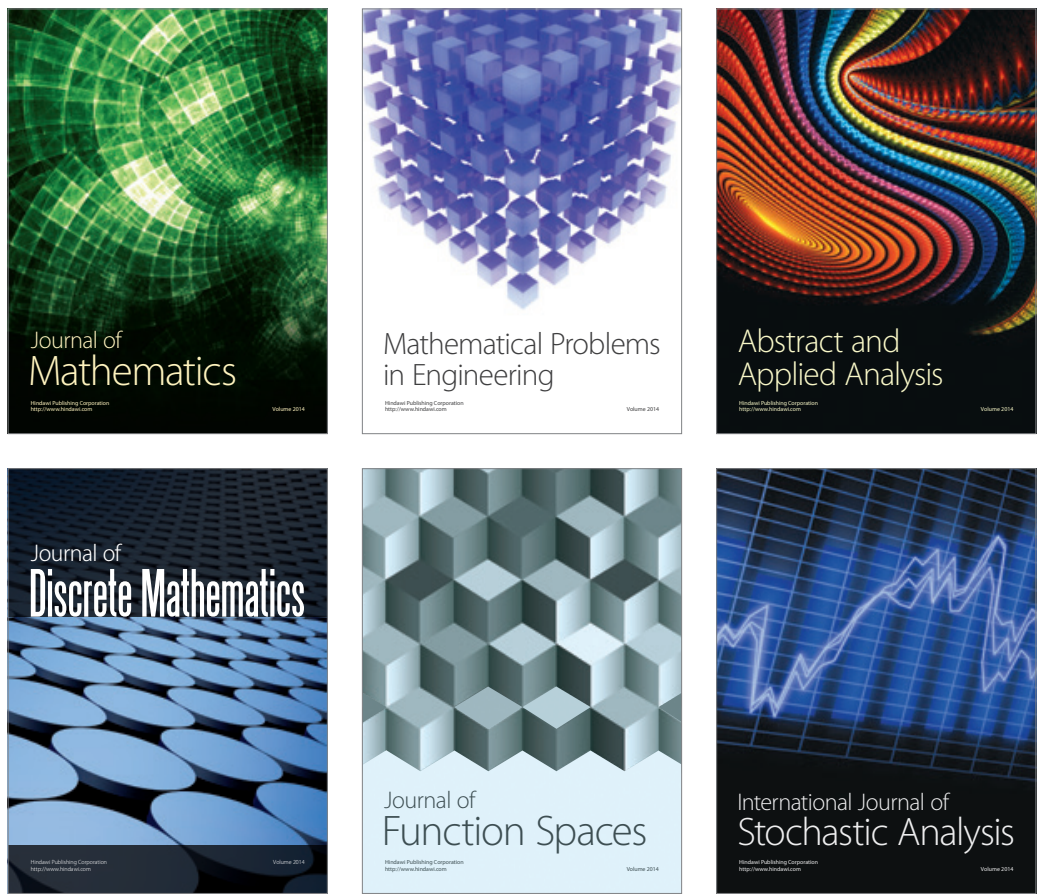

Journal of

Function Spaces

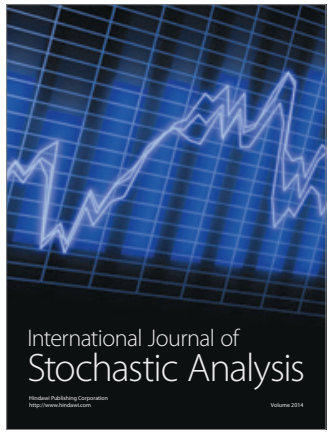

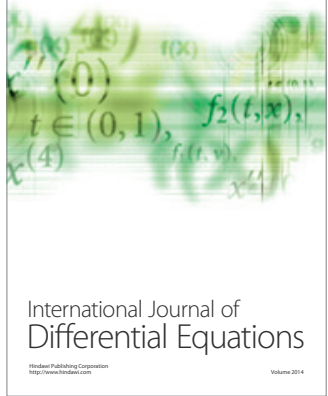
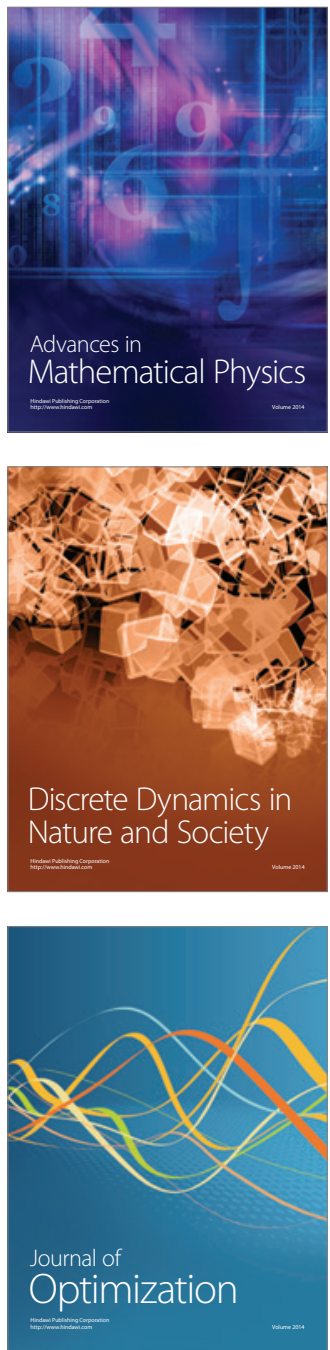